\title{
Jet Quenching in Relativistic Heavy-Ion Collisions
}

\author{
F. Canedo ${ }^{a, *}$ L. Campos, ${ }^{a}$ M. G. Munhoz, ${ }^{a}$ J. Noronha-Hostler ${ }^{b}$ and J. \\ Noronha $^{b}$ \\ ${ }^{a}$ Instituto de Física, Universidade de São Paulo, C.P. 66318, 05315-970 São Paulo, SP, Brazil \\ ${ }^{b}$ University of Illinois at Urbana Champaign, IL 61801, USA \\ E-mail: fabio.canedo@usp.br, leonardo.barreto.campos@usp.br, \\ munhoz@if.usp.br, jn0508@illinois.edu, jnorhos@illinois.edu
}

In this work, we have studied the influence of realistic modeling of the background in Relativistic Heavy-Ion collisions on Jet Quenching phenomena. We have used JEWEL to simulate the medium modified parton shower and coupled it with vUSP-hydro. For the initial conditions, we have studied both the effects of $T_{R} E N T o$ and MC-KLN. We have studied the influence of these models on jet shape observables and also on $v_{2}$. We have found no modification of the jet shape observables. We have also found that the implementation of the realistic hydrodynamical background significantly changes the $v_{2}$.

HardProbes 2020

1-6 June 2020

Austin, Texas

${ }^{*}$ Speaker 


\section{Introduction}

The modification of jet observables in Heavy-Ion collisions is an important tool to extract information about the Quark-Gluon Plasma (QGP)[9]. In this work, we have analyzed the influence of a realistic medium on a jet and compared it with a jet when it is simulated in an ideal environment. To this end, we have used JEWEL[1-3] and coupled it vUSP-hydro[4-6], a hydrodynamical code used to simulate the evolution of the QGP, we have also tested the modification of the Initial Conditions (ICs) alone. We have studied both the effects of $T_{R} E N T o[7]$ and MC-KLN[8] coupled with an ideal Bjorken longitudinal expansion. In section 2, we give a brief description of the models used in this work. In section 3 we present results obtained in this framework and, in section 4 we discuss come conclusions drawn from these results.

\section{Method}

JEWEL, in its default version, is coupled with an ideal and smooth medium, subject to a longitudinal expansion only[2]. In this work, we have generated more sophisticated medium profiles using both IC and hydrodynamical models. We have then supplied JEWEL with a temperature profile $T(\tau, x, y)$ to evolve the partonic shower. For details of this implementation, please refer to [10]. Table 1 summarizes the scenarios explored in this work.

\begin{tabular}{|c|c|c|}
\hline \multicolumn{3}{|c|}{ Scenarios } \\
\hline & Initial conditions & Evolution \\
\hline Glauber+Bjorken & Glauber & \multirow{3}{*}{ Bjorken Expansion } \\
\hline $\mathrm{T}_{\mathrm{R}} \mathrm{ENTo}+$ Bjorken & $\mathrm{T}_{\mathrm{R}}$ ENTo & \\
\hline MC-KLN+Bjorken & MC-KLN & \\
\hline MC-KLN +v-USPhydro & MC-KLN & \multirow{2}{*}{$2+1 \mathrm{v}$-USPhydro code } \\
\hline $\mathrm{T}_{\mathrm{R}}$ ENTo +v-USPhydro & $\overline{T_{R} \text { ENTo }}$ & \\
\hline
\end{tabular}

Table 1: Scenarios simulated with different ICs and hydro evolution.

To analyze the simulations, we have used both Rivet[11] and FastJet[12] packages.

\section{Results}

Figure 1 displays the results for the jet shape observables. In Figure 1a, we can see the results for girth. The simulations show no modification for this observable within the statistics generated in the simulations. Results for jet mass and $p_{T}^{D}$, displayed in Figures $1 \mathrm{~b}$ and $1 \mathrm{c}$, present similar behavior.

In Figure 2, we can see the result for the jet azimuthal anisotropy, or $v_{2}$. This observable is commonly associated with the initial geometry of the energy density distribution of the QGP. We can see that different ICs are not enough to obtain values for $v_{2}$ greater than zero. Only when we couple JEWEL with vUSP-hydro does $v_{2}$ rises. 


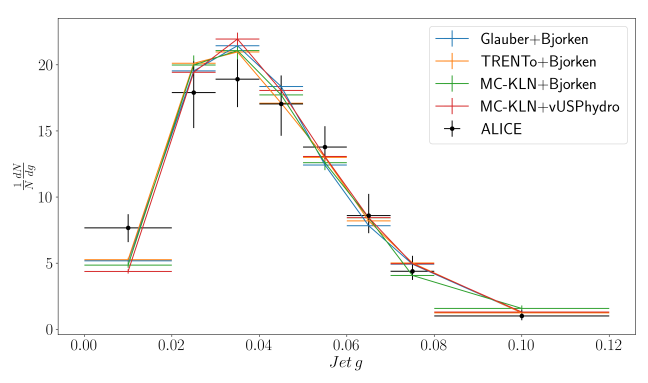

(a) Jet girth

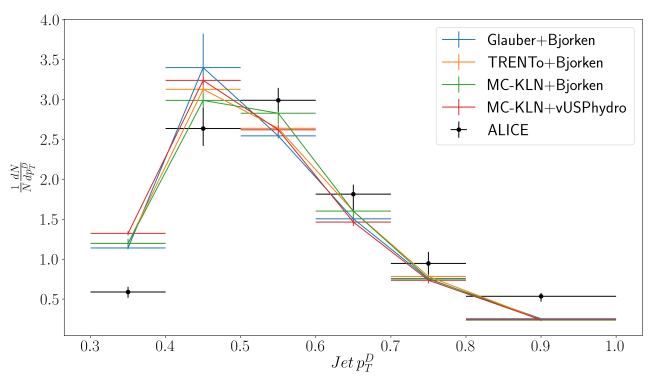

(b) $p_{T}^{D}$

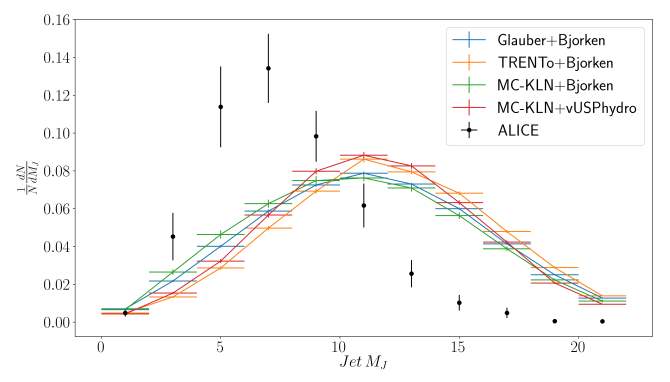

(c) Jet Mass

Figure 1: Results for shape variables of jets calculated with the anti-kt $(R=0.4)$ algorithm. The experimental data were obtained from $[13,14]$

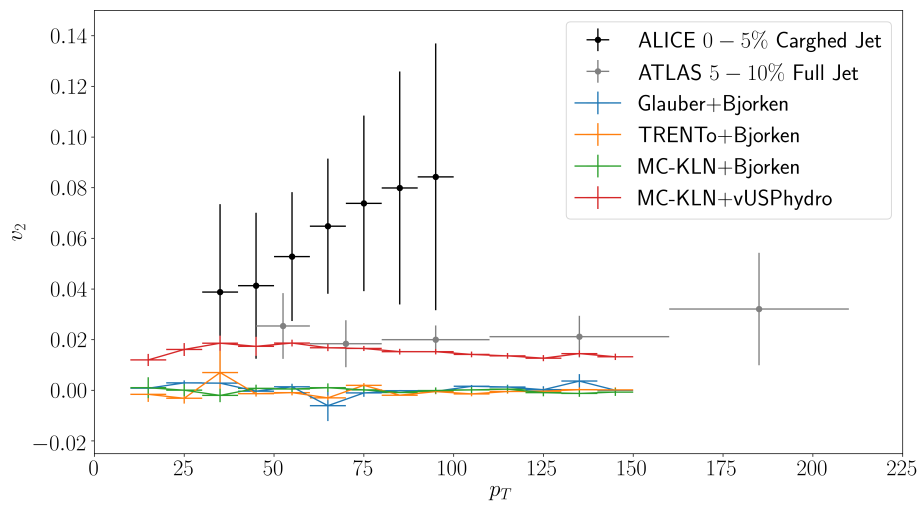

Figure 2: $v_{2}$ results. The experimental data was taken from $[15,16]$

\section{Conclusions}

We can see from the results showed in section 3. From these results, we can see that the temperature profile alone is not enough to explain the jet mass problem in JEWEL. A more appropriate way of dealing with the recoils is probably necessary. We can also see that the $v_{2}$ behavior changes significantly due to the implementation of a realistic medium evolution. 


\section{References}

[1] K.C. Zapp, JEWEL 2.0.0 - Directions for Use, The European Physical Journal C 74 (2014) .

[2] K.C. Zapp, F. Krauss and U.A. Wiedemann, A perturbative framework for jet quenching, Journal of High Energy Physics 2013 (2013) .

[3] K.C. Zapp, J. Stachel and U.A. Wiedemann, A local Monte Carlo framework for coherent QCD parton energy loss, Journal of High Energy Physics 2011 (2011).

[4] J. Noronha-Hostler, G.S. Denicol, J. Noronha, R.P.G. Andrade and F. Grassi, Bulk Viscosity Effects in Event-by-Event Relativistic Hydrodynamics, Physical Review C 88 (2013) .

[5] J. Noronha-Hostler, J. Noronha and F. Grassi, Bulk viscosity-driven suppression of shear viscosity effects on the flow harmonics at RHIC, Phys. Rev. C 90 (2014) 034907.

[6] J. Noronha-Hostler, B. Betz, J. Noronha and M. Gyulassy, Event-by-event hydrodynamics + jet energy loss: A solution to the $R_{A A} \otimes v_{2}$ puzzle, Physical Review Letters 116 (2016) .

[7] J.S. Moreland, J.E. Bernhard and S.A. Bass, Alternative ansatz to wounded nucleon and binary collision scaling in high-energy nuclear collisions, Phys. Rev. C 92 (2015) 011901.

[8] H.-J. Drescher and Y. Nara, Effects of fluctuations on the initial eccentricity from the Color Glass Condensate in heavy ion collisions, Phys. Rev. C 75 (2007) 034905.

[9] M. Connors, C. Nattrass, R. Reed and S. Salur, Review of Jet Measurements in Heavy Ion Collisions, arXiv:1705.01974 [nucl-ex] (2017) .

[10] F. Canedo, Study of Jet Quenching in Relativistic Heavy-Ion Collisions, Ph.D. thesis, May, 2020.

[11] A. Buckley, J. Butterworth, D. Grellscheid, H. Hoeth, L. Lonnblad, J. Monk et al., Rivet user manual, arXiv:1003.0694 [hep-ph] (2013) .

[12] M. Cacciari, G.P. Salam and G. Soyez, FastJet user manual: (for version 3.0.2), Eur. Phys. J. C 72 (2012) 1896.

[13] ALICE, Medium modification of the shape of small-radius jets in central Pb-Pb collisions at $\sqrt{s_{\mathrm{NN}}}=2.76 \mathrm{TeV}$, arXiv:1807.06854 [hep-ex, physics:nucl-ex] (2018).

[14] ALICE, First measurement of jet mass in Pb-Pb and $p$-Pb collisions at the LHC, Physics Letters B 776 (2018) 249.

[15] ALICE, Azimuthal anisotropy of charged jet production in $\sqrt{s_{\mathrm{NN}}}=2.76 \mathrm{TeV} \mathrm{Pb-Pb}$ collisions, Physics Letters B 753 (2016) 511.

[16] ATLAS, Measurement of the Azimuthal Angle Dependence of Inclusive Jet Yields in $P b+P b$ Collisions at $\sqrt{s_{N N}}=2.76 \mathrm{TeV}$ with the ATLAS detector, Phys. Rev. Lett. 111 (2013) 152301. 